\title{
Theory of Polymer Chains in Poor Solvent: Single-Chain Structure, Solution Thermodynamics and $\Theta$ Point
}

\author{
Rui Wang and Zhen-Gang Wang \\ Division of Chemistry and Chemical Engineering, \\ California Institute of Technology, Pasadena, CA 91125, USA
}

\begin{abstract}
Using the language of the Flory $\chi$ parameter, we develop a theory that unifies the treatment of the single-chain structure and the solution thermodynamics of polymers in poor solvents. The structure of a globule and its melting thermodynamics is examined using the self-consistent filed theory. Our results show that the chain conformation involves three states prior to the globule-to-coil transition: the fully-collapsed globule, the swollen globule and the molten globule, which are distinguished by the core density and the interfacial thickness. By examining the chain-length dependence of the melting of the swollen globule, we find universal scaling behavior in the chain properties near the $\Theta$ point. The information of density profile and free energy of the globule is used in the dilute solution thermodynamics to study the phase equilibrium of polymer solution. Our results show different scaling behavior of the solubility of polymers in the dilute solution compared to the F-H theory, both in the $\chi$ dependence and the chain-length dependence. From the perspectives of single chain structure and solution thermodynamics, our results verifies the consistency of the $\Theta$ point defined by different criteria in the limit of infinite chain length: the disappearance of the second viral coefficient, the abrupt change in chain size and the critical point in the phase diagram of the polymer solution. Our results show $\chi_{\Theta}=0.5$ (for the case of equal monomer and solvent volume), which coincides with the value predicted from the F-H theory.
\end{abstract}

\section{INTRODUCTION}

Flory-Huggins (F-H) theory is the cornerstone for polymer solution thermodynamics $\underline{1}$. The theory assumes random mixing of ideal chains with solvent molecules, and describes the polymer-solvent interaction by a phenomenological parameter $\chi$. Above the critical $\chi$ (or equivalently below the critical temperature), the F-H theory predicts phase separation of a polymer solution into a polymer-concentrated phase and polymer-dilute phase. From the F-H theory, des Cloizeaux and Jannink ${ }^{2}$ found that the equilibrium polymer concentration in the dilute phase well below the critical point scales as $\ln \phi \sim-\left(\chi-\frac{1}{2}\right)^{2} N$. The critical $\chi$ for polymer chains of length $N$ is given by $\frac{1}{2}+N^{-1 / 2}$, and has the asymptotic value of $\frac{1}{2}$ as $N$ approaches infinity. It is accepted that, in the limit of infinite chain length, the critical point for the phase separation of the polymer solution coincides with the $\Theta$ point for the coil-to-globule transition of a single chain $\underline{3}^{-\underline{7}}$. Thus, $\chi_{\Theta}=\frac{1}{2}$ in the framework of $\mathrm{F}-\mathrm{H}$ theory in the limit of infinite chain length.

The phase diagram calculated by the F-H theory shows poor agreement with experiments and simulation for the dilute phase and near the critical point: the F-H theory predicts a much lower equilibrium polymer concentration and a lower critical $\chi^{\underline{\underline{8}}} \underline{-12}$. An obvious fact that is not captured by the F-H theory is the large localized density fluctuation in the dilute solution. The instantaneous picture of the solution has much higher polymer density where the chains are located and pure solvents elsewhere, which is significantly different from the random-mixing picture envisioned in the F-H theory. For polymers in poor solvents, such localized density fluctuation takes the form of single-chain globules and multichain clusters 13 . By accounting for the large localized fluctuation, we showed in previous work ${ }^{14}$ that the solubility of the polymer in the poor solvent is enhanced by several orders of magnitude relative to the prediction of the F-H theory - the logarithm of the solubility scales with $N^{2 / 3}$ rather than $N$ as predicted from the F-H theory. The critical $\chi$ in the phase diagram for polymers of finite chain length was also shown to be pushed to higher values than $\frac{1}{2}+N^{-1 / 2}$, consistent with computer simulation and experiment results.

Another important fact not reflected in the F-H theory is the change in chain conformation according to different solvent qualities ${ }^{15}-23$. With increasing $\chi$, an isolated polymer chain will undergo the coil-to-globule transition from the swollen coil $\left(R_{g} \sim N^{3 / 5}\right)$ in good solvent to the ideal coil $\left(R_{g} \sim N^{1 / 2}\right)$ at the $\Theta$ condition, and further to the collapsed globule $\left(R_{g} \sim N^{1 / 3}\right)$ as the solvent becomes poorer. The coil-to-globule transition is a unique property of macromolecules that is different from the small molecules: even one macromolecule can form a mesoscopic phase $\frac{13,24-26}{2}$. It is a fundamental problem relevant to many interesting phenomena, such as protein folding 27 , DNA packing 28 , gel network collapse ${ }^{29}$, and interpolymer complexation $\stackrel{30}{\underline{w}}$. The coil-to-globule transition of a flexible polymer is of second-order 25.31 : in the limit of infinite chain length, there is a well-defined transition temperature known as the $\Theta$ point; whereas for the chain of finite length, the transition occurs within the $\Theta$ region of the width proportional to $N^{-1 / 2}$. De Gennes 31 pointed out that the $\Theta$ point is a tricritical point, so mean-field theory can be applied except for logarithmic corrections 32,33 . For polymer chains with sufficient stiffness, the coil-to-globule transition is predicted to become first-order ${ }^{23,34}$.

Given that the F-H theory provides a poor description 
of polymers in poor solvent, on consideration of both the over-simplified physical picture in the theory and the demonstrated inconsistency with experiments and simulation, it is natural to question the validity of the $\Theta$ value of $\chi$ predicted by the $\mathrm{F}-\mathrm{H}$ theory. The $\chi$ parameter is a general language for describing single chains, polymer solutions, blends and block copolymers. The value of $\chi_{\Theta}$ is crucially important in polymer science and engineering, because it serves as the benchmark to categorize different polymer-solvent pairs. However, existing theories for coil-to-globule transition are usually couched in the language of either the viral coefficients $34-37$ or the expansion factor ${ }^{31.38,39}$, which makes it difficult to directly connect the single chain behavior to the solution properties. Furthermore, the second virial coefficient used in the study of the single-chain behavior in previous work was obtained from the F-H free energy of the homogeneous bulk phase; the connection between this second virial coefficient and the effective two-body interaction at the level of the single-chain Hamiltonian has not be elucidated. In addition, the consistency of the $\Theta$ point defined by the different criteria concerning different properties - the second viral coefficient in dilute polymer solution, the singlechain conformation, and the critical point in the phase diagram of polymer solution in the limit of infinite chain length - has not been theoretically verified. On the other hand, computer simulation of the $\Theta$ point is challenging due to the limitation of chain length ${ }^{22,40,41}$ and numerical accuracy. On the experimental side, there are ambiguities in the structure of single-chain globule. For example, by measuring the temperature dependence of the chain size for an isolated polymer in aqueous solution, $\mathrm{Wu}$ and Wang 17,18 suggested that the chain conformation before the globule-to-coil transition involves two globule states: a fully-collapsed globule and a molten globule, where, in their words, quantitative description of the molten globule is still a challenge. They also found it surprising that a fully-collapsed globule still contains $66 \%$ solvent inside its volume.

The globule state of polymers has been theoretically studied extensively by Grosberg and coworkers 13.34-37,42 . Using the Lifshitz theory $\stackrel{25}{ }$, these authors have systematically elucidated the globule structure, globule-tocoil transition, globule-globule interaction and the phase equilibrium in dilute solution. The Lifshitz theory assumes ground-state dominance and uses a virial expansion of the local interactions. For both these two assumptions to be satisfied, the globule must on one hand have a sharp interface and on the other hand have low monomer volume fraction in the core. The combination of these two assumptions excludes a significant portion of the parameter space in the globule state. In addition, the globule structure in a poor solvent and the melting thermodynamics of a globule near the $\Theta$ point are studied by using two different theoretical formulations, the former by using the Lifshitz theory and the later by using Flory expansion factor. To complete the knowledge on the globule state of polymers, it is desirable to develop a theory that can describe the structural change of a globule in the full parameter space of the poor solvent, and can unify the globule structure and the globule melting in a single theoretical framework. In this regard, we note that Ref. $\underline{\underline{42}}$ showed that the expansion factor calculated using the F-H free energy has a large discrepancy with that obtained using the virial expansion. However, the density profile of the globule and the surface energy or its connection to solubility were not examined.

In an earlier paper, we developed a new theory in the language of the F-H $\chi$ parameter that can unify the description of the single chain structure and the solution thermodynamics for polymers in poor solvent $\underline{14}$. There, we focused on the equilibrium cluster distribution, the solubility limit, and nucleation in the supersaturated state. In the current work, we apply the theory to examine in detail the structure and properties of a single-chain globule. In our theory, we employ the full self-consistent field theory (SCFT) and treat the solution as incompressible, thus allowing us to describe both a high-density globule and melting of the globule as the $\Theta$ point is approached. From the chain-length dependence of globule melting characteristics, we investigate the scaling behavior and determine the value of $\chi_{\Theta}$ by extrapolating to the limit of infinite chain length. Because our theory is capable of describing the phase equilibrium in dilute polymer solutions, we also determine $\chi_{\Theta}$ from the solubility of polymers based on the intrinsic connection between the single chain structure and solution property.

\section{THEORY}

The system of polymers in poor solvent is a collection of globules and clusters. We study this structure of large localized density fluctuation by focusing on a subvolume of the entire solution that contains only one globule or one multi-chain cluster $14,43,44$. The density profile and free energy of the globule and clusters are obtained by applying self-consistent field theory (SCFT) in the subvolume. This information is then used in the framework of dilute solution thermodynamics to reconstruct the solution behavior of the full system. To distinguish between the composition within a single globule/cluster and the overall bulk composition in the solution, we use the notation $\rho$ and $\phi$, respectively, to denote the volume fraction of polymer in the globule/cluster and in the solution.

\section{A. Self-consistent Field Theory for an isolated globule/cluster}

We consider a subvolume $V$ consisting of $m$ polymer chains and $n$ solvent molecules. $m=1$ specifies the single-chain globule. We treat the subvolume as a semicanonical ensemble: the number of polymers in the subvolume is fixed, whereas the solvent is connected with a reservoir of pure solvent outside that maintains chemical 
potential $\mu_{s}$. (Here $\mu_{s}$ is defined relative to that of the pure solvent.) The polymers are assumed to be Gaussian chains of $N$ Kuhn segments (with Kuhn length $b$ ). For simplicity, the volume of the solvent molecule and the chain segment are assumed to be the same $v$. The local polymer-solvent interaction is described by the Flory $\chi$ parameter, and the solution is treated as incompressible.

The semi-canonical partition function can be written as:

$$
\begin{array}{r}
\Xi=\sum_{n=0}^{\infty} \frac{e^{\beta \mu_{s} n}}{m ! n ! v^{N m+n}} \prod_{j=1}^{m} \int \hat{D}\left\{\mathbf{R}_{j}\right\} \prod_{k=1}^{n} \int d \mathbf{r}_{k} \\
\quad \delta\left(\hat{\rho}_{p}+\hat{\rho}_{s}-1\right) \exp \left[-\frac{\chi}{v} \int d \mathbf{r} \hat{\rho}_{p}(\mathbf{r}) \hat{\rho}_{s}(\mathbf{r})\right]
\end{array}
$$

where $\int \hat{D}\left\{\mathbf{R}_{j}\right\}$ denotes integration over all chain configurations weighted by the Gaussian-chain statistics, and $\int d \mathbf{r}_{k}$ denotes integration over the solvent degrees of freedom. $\hat{\rho}_{s}(\mathbf{r})$ and $\hat{\rho}_{p}(\mathbf{r})$ are the local instantaneous volume fraction of solvent and polymer, respectively. The partition function Eq. 1 cannot be exactly evaluated due to both the energetic interaction and the incompressibility constraint. We make the self-consistent field (SCF) approximation, which involves (1) decoupling the interacting system into a noninteracting chains in fluctuating fields by a identity transformation using functional integration over the fluctuating fields, (2) replacing the functional integration over the fluctuating fields by the saddle point approximation. For systematic derivation and numerical details we refer readers to the standard literature $\stackrel{45,46}{ }$. The free energy of the system is then:

$$
\begin{aligned}
\beta F_{m}= & \int d \mathbf{r} \frac{1}{v}\left[\chi \rho_{p}\left(1-\rho_{p}\right)-\omega_{p} \rho_{p}-\omega_{s}\left(1-\rho_{p}\right)\right] \\
& -m \ln Q_{p}-e^{\beta \mu_{s}} Q_{s}+\ln (m !)
\end{aligned}
$$

$Q_{p}$ is the single-chain partition function in the field $\omega_{p}$, given by $Q_{p}=\frac{1}{v} \int d \mathbf{r} q(\mathbf{r}, N)$, where $q(\mathbf{r}, \tau)$ is the chain propagator determined by the diffusion equation:

$$
\left[\frac{\partial}{\partial \tau}-\frac{b^{2}}{6} \nabla_{\mathbf{r}}^{2}+\omega_{p}\right] q(\mathbf{r}, \tau)=0
$$

with initial condition $q(\mathbf{r}, 0)=1$. $Q_{s}$ is the single particle partition function of solvent in the field $\omega_{s}$, given by $Q_{s}=\frac{1}{v} \int d \mathbf{r} \exp \left(-\omega_{s}\right)$. The density profile $\phi_{p}(\mathbf{r})$ and the fields $\omega_{p}(\mathbf{r})$ and $\omega_{s}(\mathbf{r})$ are determined by the following self-consistent equations:

$$
\begin{gathered}
\omega_{p}(\mathbf{r})-\omega_{s}(\mathbf{r})=\chi\left[1-2 \rho_{p}(\mathbf{r})\right] \\
\rho_{p}(\mathbf{r})=\frac{m}{Q_{p}} \int_{0}^{N} d \tau q(\mathbf{r}, \tau) q(\mathbf{r}, N-\tau) \\
1-\rho_{p}(\mathbf{r})=e^{\beta \mu_{s}} \exp \left(-\omega_{s}(\mathbf{r})\right)
\end{gathered}
$$

By solving equations 3 and 4 iteratively $\underline{47}$, we obtain the equilibrium density profile and free energy of a globule/cluster in the subvolume.

\section{B. Effective Two-body Interaction}

If the polymer density in the subvolume is low, it is convenient to integrate over the solvent degrees of freedom, which leads to effective interaction between two polymer segments. Applying the identity transformation for $\hat{\rho}_{s}$ together with local incompressibility, we recast the partition function in Eq. 1 into:

$$
\begin{aligned}
\Xi= & \frac{1}{m ! v^{N m}} \int D \omega_{s} \prod_{j=1}^{m} \int \hat{D}\left\{\mathbf{R}_{j}\right\} \\
& \exp \left[-\int d \mathbf{r} \frac{1}{v}\left[\chi \hat{\rho}_{p}\left(1-\hat{\rho}_{p}\right)-\omega_{s}\left(1-\hat{\rho}_{p}\right)\right]+e^{\beta \mu_{s}} Q_{s}\right]
\end{aligned}
$$

Because of the high volume fraction of the solvent, the fluctuation of the field $\omega_{s}$ is small, and the functional integration over $\omega_{s}$ in Eq 5 can be replaced by the saddle point value, which yields

$$
\Xi=\frac{1}{m ! v^{N m}} \prod_{j=1}^{m} \int \hat{D}\left\{\mathbf{R}_{j}\right\} \exp [-\beta H]
$$

where $H$ is the Hamiltonian in the form of

$$
\begin{gathered}
\beta H=\int d \mathbf{r} \frac{1}{v}\left[\chi \hat{\rho}_{p}\left(1-\hat{\rho}_{p}\right)+\left(1-\hat{\rho}_{p}\right)\left(\ln \left(1-\hat{\rho}_{p}\right)-1\right)\right] \\
-\frac{\beta \mu_{s}}{v}\left(1-\hat{\rho}_{p}\right)
\end{gathered}
$$

Taking the reference such that $H$ is zero in the reservoir of pure solvent outside the subvolume, we obtain $\beta \mu_{s}=-1$. Eq 6 can then be written in the form of viral expansion in terms of instantaneous volume fraction of polymer segments as

$$
\beta H=\int d \mathbf{r} \frac{1}{v}\left[(\chi-1) \hat{\rho}_{p}+\left(\frac{1}{2}-\chi\right) \hat{\rho}_{p}^{2}+\frac{1}{6} \hat{\rho}_{p}^{3}+\cdots\right]
$$

from which we identify the effective two-body interaction as $\frac{1}{2}-\chi$. Although this is the identical expression of the effective two-body interaction commonly used in the literature, the correspondence between the effective twobody interaction and the $\mathrm{F}-\mathrm{H} \chi$ parameter in the previous theories $1, \underline{3}, \underline{13}$ was constructed for the homogeneous, bulk polymer solution; to our knowledge, this connection has not been established at the level of single-chain Hamiltonian. Here we have provided an explicit demonstration, based on a saddle-point approximation which amounts to neglecting the concentration fluctuation of the solvent, that the expression is valid at the single-chain level. If one further includes the short-length-scale concentration fluctuations, the $\chi$ parameter should more properly be interpreted as the effective interaction parameter instead of the bare interaction parameter ${ }^{48,49}$. Renormalization group theory ${ }^{\underline{4}}$ showed that the second viral coefficient of the polymer solution has corrections from the threebody interaction. Grosberg and Kuznetsov ${ }^{34}$ pointed out 
that this correction vanishes in the limit of infinite chain length. Therefore, both the effective two-body interaction and the second viral coefficient vanish at $\chi_{\Theta}=\frac{1}{2}$ for infinitely long chain.

\section{Phase Equilibrium}

The density profile and free energy of the globule and clusters obtained from SCFT can be included into the framework of dilute solution thermodynamics to reconstruct the bulk dilute polymer solution. The free energy density of the entire solution with volume $V_{t}$, including the translational entropy of clusters, can be written as

$$
\beta F / V_{t}=\sum_{m=1}^{\infty}\left[C_{m} \beta F_{m}+C_{m}\left(\ln \phi_{m}-1\right)\right]
$$

where $C_{m}$ and $\phi_{m}$ are respectively the concentration and volume fraction of the cluster with association number $m$ (called $m$-cluster henceforth) in the solution. $F_{m}$ is the free energy of the $m$-cluster calculated by SCFT (obtained as an excess free energy in the subvolume with respect to the pure solvent). In Eq 8, we ignore the interaction between different clusters on the assumption that the solution is sufficiently dilute. The equilibrium concentration of $m$-clusters can be obtained by minimization of the free energy density in Eq 8 subject to fixed total polymer concentration $\sum_{m=1}^{\infty} m C_{m}$, which results in the following cluster distribution:

$$
\phi_{m}=\left(\phi_{1}\right)^{m} \exp \left(-\beta \Delta F_{m}\right)
$$

where $\Delta F_{m} \equiv F_{m}-m F_{1}$ is the free energy of formation of the $m$-cluster from $m$ single chain globule.

To study the coexistence between the polymer-poor phase and the polymer-rich phase, we use Eq 8 with Eq. 9 to account for the large localized density fluctuation in the polymer-poor solution, whereas the polymer-rich phase is described by the F-H theory. The phase boundary is determined by the respective equality of the chemical potential of the polymer and the solvent in the two coexisting phases, which results in

$$
\begin{aligned}
-\sum_{m=1}^{\infty} \frac{\phi_{m}}{m N}= & \left(1-\frac{1}{N}\right) \phi_{H}+\ln \left(1-\phi_{H}\right)+\chi \phi_{H}^{2} \\
\beta F_{1}+\ln \phi_{1}= & \ln \phi_{H}-1+(1-N)\left(1-\phi_{H}\right) \\
& +\chi N\left(1-\phi_{H}\right)^{2}
\end{aligned}
$$

where $\phi_{H}$ is the equilibrium volume fraction of polymers in the polymer-rich phase and $\phi_{m}$ is the equilibrium volume fraction of the $m$-cluster in the dilute phase given by Eq 9 . The total volume fraction of polymers in the dilute phase, $\phi_{L}$, is given by $\phi_{L}=\sum_{m=1}^{\infty} \phi_{m}$.

\section{GLOBULE STRUCTURE}

In the poor solvent, a single polymer chain adopts a compact globular structure due to the unfavorable inter- action between the polymer segments and the solvent. The increase in local segment density is countered by excluded volume (modeled by the incompressibility in our theory) which prevents the globule from collapsing into unbounded high density. Figure 1 shows the density profile of a globule for different values of $\chi$ calculated by SCFT. Clearly the globule can be divided into a core region with uniform density (denoted by $\rho_{0}$ ) and a surface region. The globule core resembles a liquid droplet that contains a number of uncorrelated parts of the chain. By taking uniform fields $\omega_{p}$ and $\omega_{s}$ in equations 2-4, we obtain the free energy in the globule core to be

$$
\beta F_{c}=\frac{N}{\rho_{0}}\left[\chi \rho_{0}\left(1-\rho_{0}\right)+\left(1-\rho_{0}\right) \ln \left(1-\rho_{0}\right)\right]
$$

which is the Flory-Huggins free energy without the translational entropy of the polymer due to the fixed center of mass. In the limit of large $N$, the Laplace pressure due to curvature can be neglected, and the polymer density of the globule core can be obtained approximately by balancing the osmotic pressure inside the globule with that of the pure solvent outside $(\approx 0)$, which yields $\rho_{0}$ as the nontrivial root of the following equation ${ }^{50}$

$$
\rho_{0}+\ln \left(1-\rho_{0}\right)+\chi \rho_{0}^{2}=0
$$

For $\chi$ close to 0.5 , Eq 12 has the symptomatic solution of $\rho_{0} \approx 3(\chi-0.5)$. As shown in Figure 1, the core density becomes lower with the decreasing $\chi$, which increases the size of the globule as a result of swelling. At the same time, the interfacial region becomes thicker and more diffuse. We see from Figure 2 that the thickness of the interfacial region scales with the Kuhn length $b$ as pointed out by Grosberg and Khokhlov $\underline{13}$ and is independent of the chain stiffness parameter $p=b^{3} / v$. Because the dependence on $p$ is relatively straightforward, in most of the subsequent discussions, we choose a specific $p$ corresponding to $v=4 \pi b^{3} / 3$.

Based on the density profile, we can define the radius of gyration $\left(R_{g}\right)$, the core radius $\left(R_{0}\right)$ and the surface diffuseness $(h)$ to characterize the globule size, which are given as:

$$
\begin{aligned}
& R_{g}=\left(\frac{\int_{0}^{\infty} r^{2} \rho(r) 4 \pi r^{2} d r}{\int_{0}^{\infty} \rho(r) 4 \pi r^{2} d r}\right)^{1 / 2} \\
& R_{0}=(3 / 5)^{1 / 2}\left[3 N v /\left(4 \pi \rho_{0}\right)\right]^{1 / 3} \\
& h=R_{g}-R_{0}
\end{aligned}
$$

$R_{0}$ is the radius of gyration for a uniform sphere with polymer density $\rho_{0}$ that contains the same total amount of chain segments as in the globule; in other words, $r=$ $(5 / 3)^{1 / 2} R_{0}$ is the location of the Gibbs dividing surface. $h$ describes the deviation of the globule from the uniform sphere, thus characterizing the diffuseness of the globule surface. It should be noted that $h$ is not a linear measure of the true thickness of the globule surface (denoted by $h_{T}$ ) within which the polymer density changes from $\rho_{0}$ 


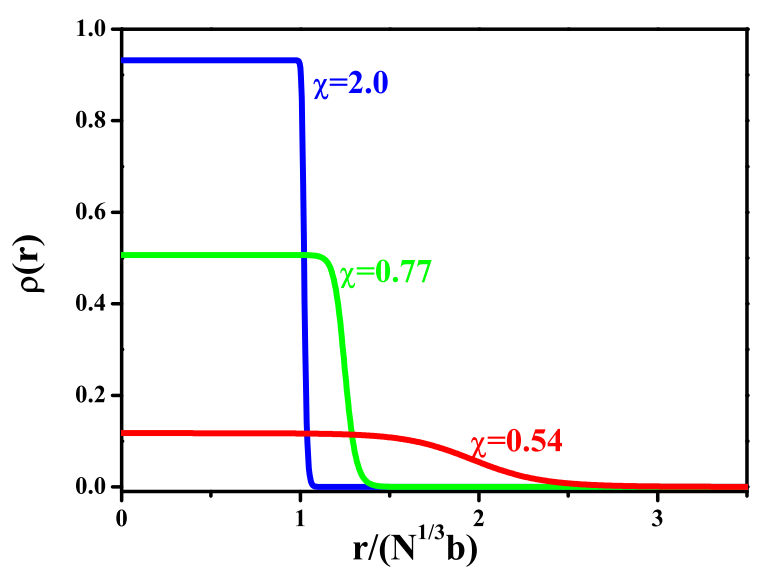

FIG. 1. Density profile of a globule for three values of $\chi$ with $N=10^{4}$ and $v=4 \pi b^{3} / 3 . \rho(r)$ is the local volume fraction of polymer with $r$ the radial axis starting from the globule center.

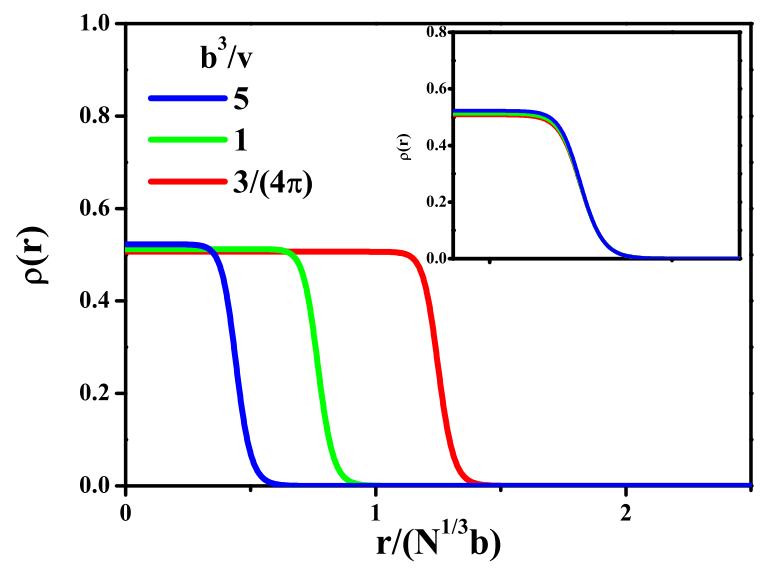

FIG. 2. Effect of the chain stiffness parameter $p=b^{3} / v$ on the density profile of a globule with the same Kuhn length. $\chi=0.77$ and $N=10^{4}$. The insert shows the overlap of the interfacial region when the density profiles are shifted.

to 0 . Dobrynin and Rubinstein $\frac{51}{1}$ pointed out that the surface thickness of the globule has a length scale of one thermal blob $h_{T} \sim b / \rho_{0}$. For a globule with a large uniform core (i.e., $h_{T} / R_{0} \ll 1$ ), it can be shown that the surface diffuseness scales as $h \sim h_{T}^{2} / R_{0} \stackrel{52}{ }$ In this work, we choose $h$ rather than $h_{T}$ to characterize the globule surface because $h$ is defined based on $R_{g}$, which is an experimentally measurable quantity.

With the definition of $R_{0}$ and $h$, we can separately examine the volume contribution and the surface contribution to the globule size. The core density and the globule size as a function of $\chi$ for polymer with $N=10^{4}$ are shown in Figure 3 . When $\chi$ is well above $0.5, R_{g}$ coincides with $R_{0}$ while $h$ remains very small and is negligible compared to $R_{0}$. The globule surface is of the thickness of one thermal blob $\left(h_{T} \sim b / \rho_{0}, h \sim b \rho_{0}^{-5 / 3} N^{-1 / 3}\right)$, and the entire globule is space-filled by $\sim \rho_{0}^{2} N$ thermal blobs. In this regime, the globule can be envisioned as a uniform sphere: the increase of the globule size as $\chi$ decreases is due to the swelling of the globule core (see Figure $3 \mathrm{~b}$ ), given by $R_{g} \approx R_{0} \sim\left(N v / \rho_{0}\right)^{1 / 3}$. As $\chi$ approaches 0.5 , $R_{g}$ increases rapidly and deviates from $R_{0}$. At the same time, $h$ increases sharply and becomes comparable to $R_{0}$. The thermal blob expands, reaching a size comparable to the whole globule. The rapid increase of the globule size in this regime indicates melting of the globule, which takes place by both the swelling of the globule core and the widening of the interface.

The $\chi$ dependence of the globule size and core density shown in Figure 3 suggests that the single chain conformation prior to the globule-to-coil transition involves three states: the fully-collapsed globule, the swollen globule and the molten globule, in the order of decreasing $\chi$. For large $\chi(\chi>1)$, the globule is fully-collapsed, with core density close to one and a sharp interface: $\rho_{0} \approx 1, h_{T} / R_{0} \sim N^{-1 / 3}$ and $h / R_{0} \sim N^{-2 / 3}$. The fullycollapsed globule can further transform to the ordered solid-globule as pointed out by Zhou et al $\stackrel{53}{\text {, which is }}$ out of the scope of this work. In the intermediate regime, where $\chi-0.5 \ll 1$ but $\left(\chi-\chi_{\Theta}\right) N^{1 / 2} \gg 1$, the globule is swollen. The core density is significantly lower than one, while the interfacial thickness is still narrow compared to the core radius: $\rho_{0}<<1, h_{T} / R_{0} \sim\left[\left(\chi-\chi_{\Theta}\right) N^{1 / 2}\right]^{-2 / 3}$, $h / R_{0} \sim\left[\left(\chi-\chi_{\Theta}\right) N^{1 / 2}\right]^{-4 / 3}$. Finally in the $\Theta$ regime, where $\left(\chi-\chi_{\Theta}\right) N^{1 / 2} \sim O(1)$ or less, the globule is molten, with a very dilute core, comparable to a Gaussian coil, and an interfacial thickness comparable to the core radius: $\rho_{0} \sim\left(\chi-\chi_{\Theta}\right) \sim N^{-1 / 2}, h_{T} / R_{0} \sim O(1)$, and $h / R_{0} \sim O(1)$.

That the globule structure may involve more than one states has been suggested before. Wu and Wang 17,18 proposed that the globule structure involves two states: the fully-collapsed globule and the molten globule, based on the consideration that the ratio between the radius of gyration and hydrodynamic radius for the fully-collapsed globule is $(3 / 5)^{1 / 2}$ whereas this ratio for the molten globule is smaller than $(3 / 5)^{1 / 2}$ (this criterion is equivalent to whether the globule has a sharp or diffuse surface). However, the "fully-collapsed" globule observed in their experiment contains $66 \%$ solvent inside the globule volume, which is more properly considered as the swollen globule in our definition. If we choose $\chi=1$ as the criterion for separating the swollen globule from the fullycollapsed globule, then the range of the swollen globule is roughly between $\chi_{\Theta}$ and $\chi=1$ for large $N$; this can correspond to a large temperature window in experiments. The swollen state for the infinitely long chain persists all the way to the $\Theta$ point, as we discuss below. 

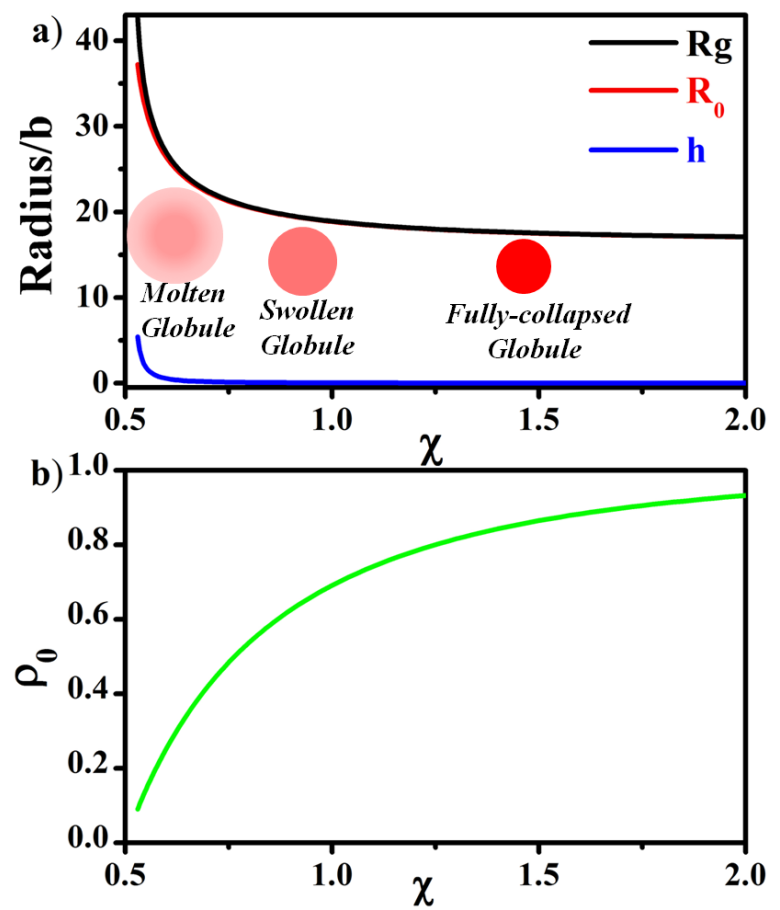

FIG. 3. a) Radius of gyration $\left(R_{g}\right)$, core radius $\left(R_{0}\right)$ and interfacial diffuseness $(h)$ and b) core density $\left(\rho_{0}\right)$ of the globule as a function of $\chi$ with $N=10^{4}$. The schematic inserted in a) represents the three states in globule structure: the fullycollapse globule, the swollen globule and the molten globule.

It is instructive to note an analogy between the swollen globule and the semidilute polymer solution in good solvent. The existence of both these states is due to the long chain length. Indeed the molten globule, the swollen globule and the fully-collapsed globule can be considered the respective analogues of the dilute, semi dilute, and concentrated solutions. Just as there is no clearcut boundary between the semidilute and concentrated solutions, the distinction between a swollen globule and fully collapse globule is not a sharp one. And just as the concentration range for the dilute regime becomes narrower as the chain length increases, so the region of the molten globule shrinks with increasing chain length. Likewise, with decreasing chain length, the window for the existence of the swollen globule shrinks, and eventually disappears for very short chains.

We should emphasize that the density profile of the globule is calculated using the flexible Gaussian chain model. If the thickness of the globule surface becomes comparable to the Kuhn length, which happens for very large $\chi$, the chain segments can become oriented by the interface. In that case, the flexible Gaussian chain is no longer a valid description and chain rigidity and local nematic order may have to be accounted for explicitly 54 . However, we find that for $\chi=1.0$ (with the core density $\left.\rho_{0}=0.7\right)$ the interfacial width is $h_{T}=5 b$ and is
$h_{T}=10 b$ for $\chi=0.77$ (with the core density $\rho_{0}=0.52$ ). The interfacial width decreases to the Kuhn length at about $\chi=2.0$, corresponding to a core density $\rho_{0}=0.93$. Therefore, there is a significant range of parameter space in which the Gaussian chain model remains valid.

\section{DETERMINING $\chi_{\Theta}$ FROM THE SINGLE-CHAIN GLOBULE}

The globule structure obtained by our SCFT calculation is valid under the condition that the surface energy of the globule is much larger than $k T$; otherwise shape fluctuation must be taken into account and the assumption of spherical symmetry becomes questionable. This condition is equivalent to the requirement that the number of thermal blobs $\rho_{0}^{2} N$ must be large, i.e., $\rho_{0}^{2} N \gg 1$. To satisfy this requirement, the globule state should be within the parameter space $\left(\chi-\chi_{\Theta}\right) N^{1 / 2} \gg 1$, i.e., the chain should be in the fully-collapsed or swollen globule. (Equation 17b provides a more quantitative Ginzburg criterion.) This requirement would at first seem to prevent us from applying the theory to the close vicinity of the $\Theta$ point. However, as the chain length increases, the width of the $\Theta$ region (in $\chi$ ) shrinks and the transition becomes increasingly sharper ${ }^{23,31.34}$. In other words, the state of the swollen globule persists closer to the $\Theta$ point. In the limit of infinitely long chain, the swollen globule persists all the way to the $\Theta$ point. Therefore, the chain length dependence of the melting of the swollen globule can be used to probe the width of the $\Theta$ region and the location of the $\Theta$ point.

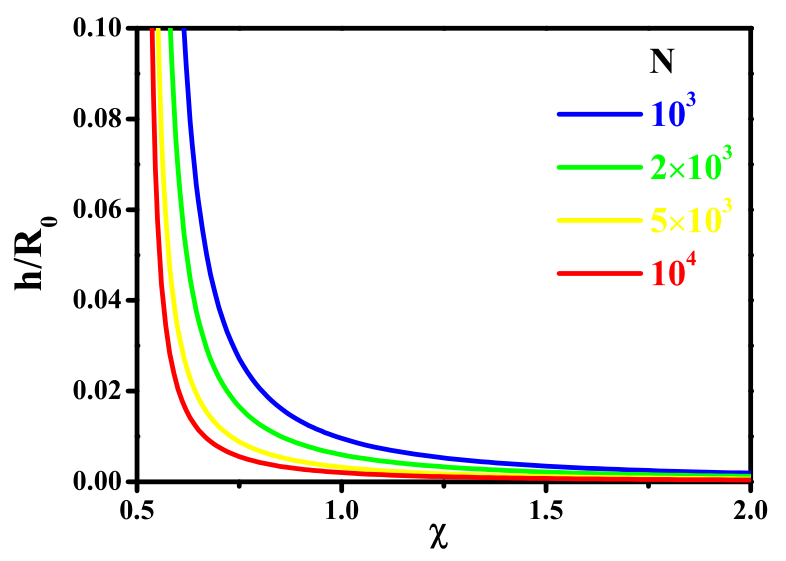

FIG. 4. The ratio between the surface diffuseness and the core radius $\left(h / R_{0}\right)$ as a function of $\chi$ for different chain length.

Figure 4 shows the ratio between the surface thickness and the core radius of the globule $\left(h / R_{0}\right)$ as a function of $\chi$ for several different chain lengths. As $\chi$ decreases close to $0.5, h / R_{0}$ exhibits a rapid rise as a result of the melting of the globule; this rise is sharper for larger $N$. 
From Figure 4, we can define the onset of the melting (denoted by $\chi_{N}(\lambda)$ ) as the value of $\chi$ at which $h / R_{0}$ reaches a threshold $\lambda . \chi_{N}(\lambda)$ thus represents the onset of the globule-to-coil transition as well as the boundary of the $\Theta$ region on the globule side. Clearly, $\chi_{N}(\lambda)$ depends on chain length. Figure 5 shows the linear relation of $\chi_{N}(\lambda)$ versus $N^{-1 / 2}$ for three different levels of $\lambda$; this linearity demonstrates that the width of the $\Theta$ region is proportional to $N^{-1 / 2}$, in agreement with the results from the scaling argument $\underline{31}$ and simulation 22 .

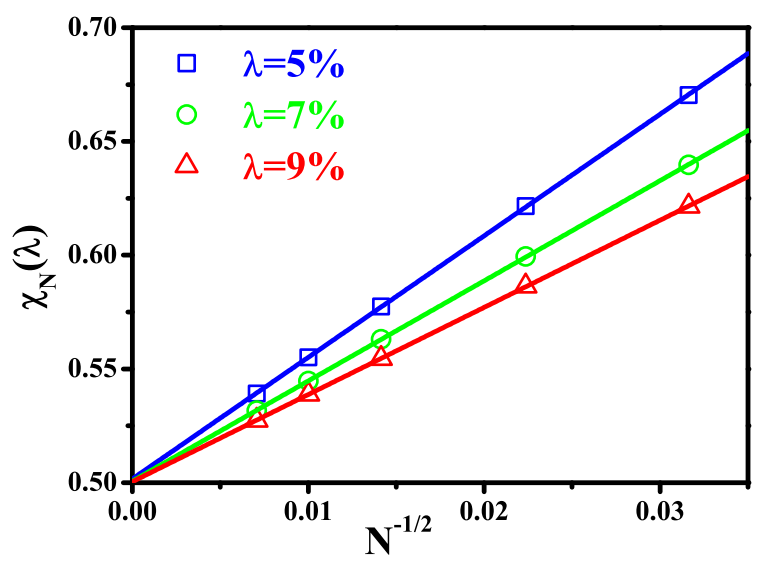

FIG. 5. The onset of globule melting $\chi_{N}(\lambda)$ plotted vs $N^{-1 / 2}$ for three different $\lambda$. Points represent the results calculated by SCFT. Straight lines are the linear fit to the points. Three lines intersect to a common point as $N \rightarrow \infty$, which yields $\chi_{\Theta}=0.5$.

As $N$ increases, $\chi_{N}(\lambda)$ for the different choices of the threshold $\lambda$ become closer, indicating that the transition becomes sharper for longer polymer chains. Extrapolating $\chi_{N}(\lambda)$ versus $N^{-1 / 2}$ to the limit of $N \rightarrow \infty, \chi_{N}(\lambda)$ for different $\lambda$ 's converge to a common point that is independent of the choice of $\lambda$, indicating that the transition is infinitely sharp as $N \rightarrow \infty$. From the perspective of single-chain conformation, $\Theta$ point is defined as the abrupt change in chain size from globule to coil for an infinitely long chain. The common intersection shown in Figure 5 yields $\chi_{\Theta}=0.50$. This value coincides with the result of F-H theory and is also consistent with the vanishing of the second viral coefficient derived in Sec II. We comment that the coincidence of $\chi_{\Theta}=0.50$ with the prediction of the F-H theory is not a trivial result, nor does it mean that the F-H theory itself is valid. Indeed, the F-H theory is a poor description of the dilute solution, including the solution near the $\Theta$ point. However, if we focus on the core of an isolated globule, it is a homogeneous region containing many uncorrelated blobs of the chain, sharing the same physical picture as the F-H theory. In the limit of the infinite chain length, the swollen globule state persists all the way to the $\Theta$ point where the effective two-body interaction vanishes.

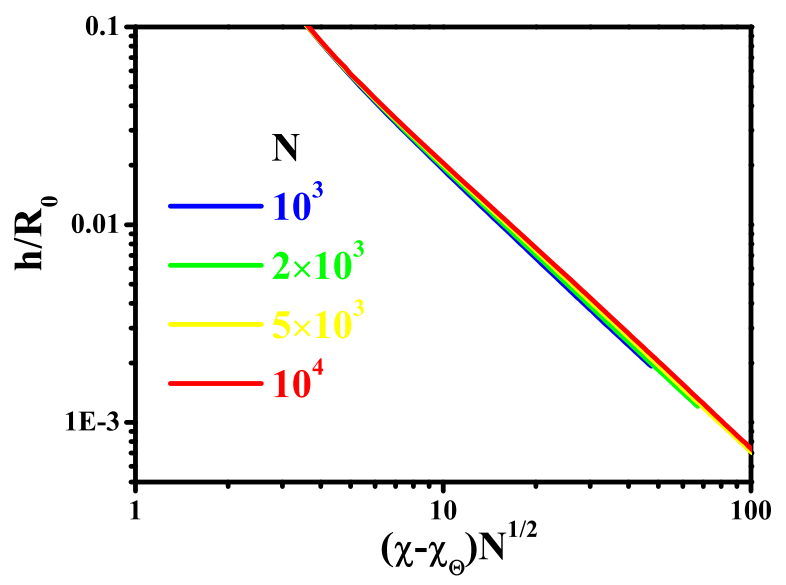

FIG. 6. Universal behavior in the scaling region. log-log plot of $h / R_{0}$ vs $\left(\chi-\chi_{\Theta}\right) N^{1 / 2}$.

Using $\chi_{\Theta}, h / R_{0}$ can now be plotted as a function of the scaling variable $\left(\chi-\chi_{\Theta}\right) N^{1 / 2}$. Figure 6 shows that $h / R_{0}$ for different chain lengths collapse onto each when plotted against $\left(\chi-\chi_{\Theta}\right) N^{1 / 2}$, confirming the universal behavior near the $\Theta$ point:

$$
h / R_{0}=f\left[\left(\chi-\chi_{\Theta}\right) N^{1 / 2}\right]
$$

where $f$ is a universal function. In the range $\left(\chi-\chi_{\Theta}\right) N^{1 / 2}>10$ (where the globule is in the swollen state with low core density), the slope of the log-log plot is -1.4 , close to the exponent $-4 / 3$ we have obtained in Sec III by using the scaling argument. The universal behavior of the single chain conformation around the $\Theta$ point, initially predicted by de Gennes ${ }^{31}$, is usually difficult to achieve in the globule state in computer simulation $\underline{22}$ due to the limitation of chain length. For short chains, the swollen globule either is not well-defined or cannot persist to a very dilute core, thus making the window for observing the scaling regime unclear or too small. Here, by applying SCFT to long chains $\left(N>10^{3}\right)$, we are able to more definitively examine this scaling regime in the globule state.

\section{DETERMINING $\chi_{\Theta}$ FROM SOLUTION THERMODYNAMICS}

The single chain structure will of course affect the solution properties. The compaction of chain segments into a globule significantly reduces the polymer-solvent contact, which leads to enhancement of the solubility of polymer in poor solvent by several orders of magnitude compared to the prediction of the F-H theory as shown in our earlier paper $\stackrel{14}{ }$. The governing equation for the 
phase equilibrium of polymers in poor solvent is given by Eq 10 in Sec IIC. In Eq 10a, the osmotic pressure for long polymer chain in the dilute solution is very small $\left(\sum_{m=1}^{\infty} \phi_{m} / m N \approx 0\right)$. Thus, from the similarity between Eq 10a and Eq 12, we obtain for the volume fraction of the polymer-rich phase,

$$
\phi_{H} \approx \rho_{0}
$$

On the other hand, based on our analysis of the globule structure in Sec III, the free energy of the single chain globule in Eq 10b can be divided into the volume contribution and the surface contribution as $F_{1}=F_{c}+F_{s}$. It can be shown that this definition is consistent with the definition based on the monomeric osmotic pressure across the interface used in Ref 13 . By substituting $F_{c}$ in the form of Eq 11 into Eq 10b, and making use of Eq 12 and Eq 15, we obtain the relation between the equilibrium volume fraction of polymers in the dilute phase and the surface energy of the globule

$$
\ln \phi_{L} \approx-\beta F_{s}+\ln \rho_{0}-\rho_{0}=-\gamma A+\ln \rho_{0}-\rho_{0}
$$

from which we identify the surface tension $\gamma$. $A=$ $(36 \pi)^{1 / 3}\left(N v / \rho_{0}\right)^{2 / 3}$ is the surface area of the globule. Noting that the natural dimension of the surface tension is $b / v$ ( $k T$ is taken to be 1$)$, a dimensionless surface tension can be defined as $\gamma v^{2 / 3}$ which thus contains an overall $p^{1 / 3}$ dependence; this is the origin of the $p^{1 / 3}$ in Eqs. $17 \mathrm{a}$ and 17b. Eq 16 indicates that, the excess free energy of a globule is due to its surface energy, and not due to (uniform) mixing of polymer segments and solvents assumed in the F-H theory. Equations 15 and 16 reveal the intrinsic connection between the macroscopic phase behavior of the polymer solution and the microscopic structure and property of the single globule. This connection also allow us to extract information of single chain globule from solution thermodynamics.

The linear relation between $\ln \phi_{L}$ and $N^{2 / 3}$ is confirmed by numerically solving Eq. 10 as shown in Figure 7. From the slope of $\ln \phi_{L}$ versus $N^{2 / 3}$, we can extract the surface tension of a single globule. Figure 8 shows that for $\chi$ much larger than $\chi_{\Theta}, \gamma$ decreases linearly with $\chi$ as $\gamma v^{2 / 3}=0.11 \chi-0.07$. This linear dependence on $\chi$ agrees with the result of Weber and Helfand for the interfacial tension of a planar interface ${ }^{55}$. As $\chi$ decreases, $\gamma$ deviates from the linear behavior and approaches zero. The disappearance of the surface tension of the globule serves as another definition of the $\Theta$ point. By extrapolating the numerical data to the limit of $\gamma=0$, Figure 8 yields $\chi_{\Theta}=0.50$, which coincides with previous results obtained from other definitions. Figure 8 also shows that, $\gamma$ follows a quadratic form $\gamma v^{2 / 3}=0.52(\chi-0.5)^{2}$ for $\chi-\chi_{\Theta} \ll 1$. The quadratic form of $\gamma$ near the $\Theta$ point is in agreement with scaling prediction by Lifshitz et al ${ }^{25}$.

In the framework of F-H theory, des Cloizeaux and Jannink obtained $\phi_{L}=\frac{3}{e}\left(\chi-\frac{1}{2}\right) \exp \left[-\frac{3}{2}\left(\chi-\frac{1}{2}\right)^{2} N\right]$. By substituting the expression of $\gamma$ and $\rho_{0}$ into Eq 16, our

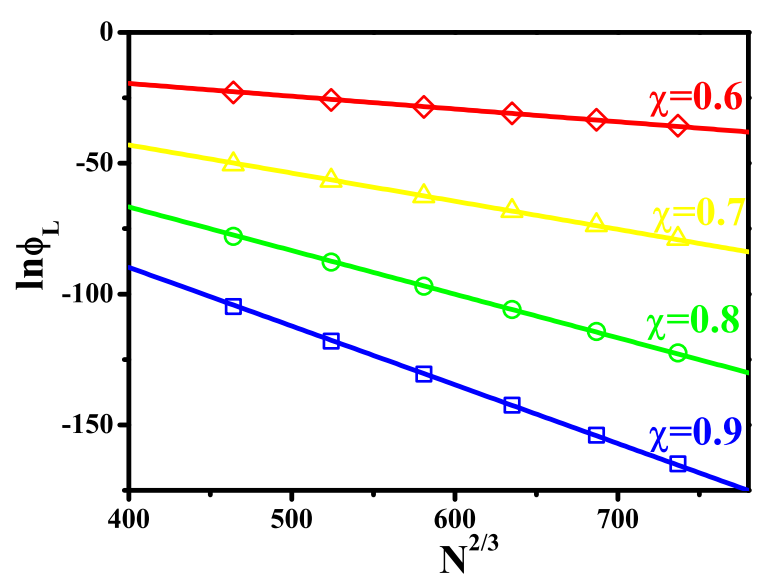

FIG. 7. Linear relation between $\ln \phi_{L}$ and $N^{2 / 3}$. Points represent the numerical results based on Eq 10. Straight lines are the linear fit of the points.

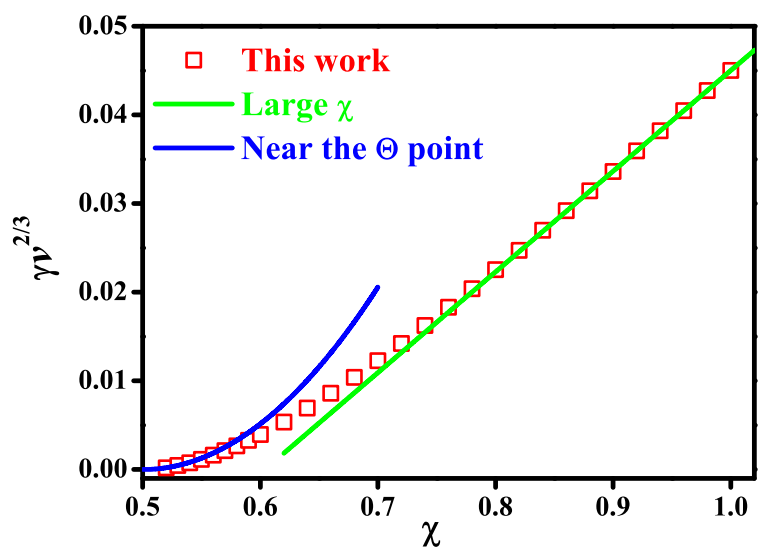

FIG. 8. Dimensionless surface tension of the globule $\gamma v^{2 / 3}$ as a function of $\chi$. Extrapolating the numerical data to $\gamma=0$ reveals $\chi_{\Theta}=0.5$. $\gamma$ is fitted linearly for large $\chi$ as $\gamma v^{2 / 3}=$ $0.11 \chi-0.07$, and fitted by a quadratic function $0.52(\chi-0.5)^{2}$ near the $\Theta$ point.

theory yields the equilibrium volume fraction of polymers in the dilute phase as

$$
\begin{aligned}
& \phi_{L}=\frac{1}{e} \exp \left[-p^{1 / 3}(0.89 \chi-0.53) N^{2 / 3}\right] \\
& \text { for large } \chi \\
& \phi_{L}=3(\chi-0.5) \exp \left[-1.93 p^{1 / 3}(\chi-0.5)^{4 / 3} N^{2 / 3}\right] \\
& \text { for } \chi-\chi_{\Theta} \ll 1
\end{aligned}
$$

where we have inserted the dependence on the stiffness parameter $p=b^{3} / v$. We note that an equation similar 
to Eq 17b has been given in Ref $\frac{13}{1}$ (Eq. 26.8). However, our Eq. 17b provides the numerical prefactor (1.93) in the scaling dependence in the exponential, which was not provided in Ref 13 . Eq. 17a is a completely new result. These equations show very different scaling behavior of the solubility of polymers in poor solvent predicted by our theory compared to the F-H theor ${ }^{2}{ }^{2}$, both in the $\chi$ dependence and in the $N$ dependence.

The exponent in Eq. 17b can be considered as a quantitative Ginzburg criterion for the validity of applying the SCFT, i.e., SCFT is applicable if $p^{1 / 3}(\chi-0.5)^{4 / 3} N^{2 / 3}>$ 1. Thus increasing the stiffness parameter $p$ allows a closer approach to the $\Theta$ point. This conclusion is consistent with results from computer simulation by Withers et al ${ }^{33}$.

From Eq 17b, we obtain the slope of the phase boundary near the $\Theta$ point as

$$
\begin{aligned}
\frac{\partial \phi_{L}}{\partial \chi}= & {\left[3-7.74 p^{1 / 3}(\chi-0.5)^{4 / 3} N^{2 / 3}\right] } \\
& \exp \left[-1.93 p^{1 / 3}(\chi-0.5)^{4 / 3} N^{2 / 3}\right]
\end{aligned}
$$

Consistent with the validity of the SCFT, Eq 18 is valid in the swollen globule state, which can persist all the way to the $\Theta$ point for infinitely long chain. In the limit of $N \rightarrow \infty, \partial \phi_{L} / \partial \chi=0$ if $\chi>0.5$. However, $\partial \phi_{L} / \partial \chi$ jumps to a finite value at $\chi=0.5$, which suggests that $\chi=0.5$ is the critical point in the phase diagram of a polymer solution in the limit of infinite chain length $\underline{56}$. This establishes the $\Theta$ point defined from the perspective of solution thermodynamics.

These results confirm that the $\Theta$ point defined as the abrupt change in chain size from globule to coil and as the critical point in the phase diagram are consistent with each other in the limit of infinite chain length. This consistency reveals the intrinsic connection between the single chain structure and its solution properties. From the perspective of solution thermodynamics our results suggest an alternative experimental determination of the $\Theta$ point from measuring the slope of $\ln \phi_{L}$ versus $N^{2 / 3}$ at different temperatures. Since this approach is based on finite polymer concentrations, it may be more easily conducted than the single-chain measurement.

\section{CONCLUSIONS}

In this work, we have presented a theory that unifies the study of the single-chain structure and solution thermodynamics for polymers in poor solvents, using the language of the Flory-Huggins parameter. Our theory captures the large localized density fluctuation in dilute polymer solutions and the change in chain conformation with the solvent quality that are missing in the F-H theory. The structure of a globule is studied by SCFT, which affords a more accurate description of the density profile of the globule and its free energy for finite chain lengths. By relaxing the assumption of the virial expansion of the local interaction as assumed in previous theories, our theory can be applied to globules with relatively high monomer density, which facilitates the calculation of the surface tension of the globule and the solubility of polymers in dilute solution for large values of $\chi$. On the other hand, by avoiding the ground state dominance approximation, the SCFT is capable to describe globules with diffuse interfaces. These advantages allow us to study the globule structure and the thermodynamics of the globule melting in a unified theoretical framework. The chainlength dependence of the globule melting provides the essential information on the approach to the $\Theta$ point.

We briefly summarize the key new results of this work. First, we show that the chain conformation involves three globular states prior to the globule-to-coil transition: the fully-collapsed globule, the swollen globule and the molten globule; this identification clarifies the ambiguity in the experimental studies of the globule structure. Second, we provide numerical verification of the universal behavior as a function of the scaling variable $\left(\chi-\chi_{\Theta}\right) N^{1 / 2}$ near the $\Theta$ point, which has not been achieved by previous computer simulation in the globule state due to the limitation of the chain length. Third, we provide new results for the solubility of the polymers in dilute solution compared to the results of des Cloizeaux and Jannink based on the F$\mathrm{H}$ theory. The large $\chi$ result is completely new while the result near the $\Theta$ point provides the missing numerical prefactor in previous work. Fourth, we demonstrate the consistency of the $\Theta$ points defined by the different criteria in the limit of infinite chain length: the disappearance of the second viral coefficient in the dilute polymer solution, the abrupt change in chain size from globule to coil and the critical point in the phase diagram of polymer solution. Fifth, we find $\chi_{\Theta}=0.5$ from all three different criteria, which coincides with the prediction of $\mathrm{F}-\mathrm{H}$ theory. Lastly, although the expression itself is known and widely used in the literature, we provide the explicit derivation that shows the effective two-body interaction in the single-chain Hamiltonian is given by $0.5-\chi$.

Although $\chi_{\Theta}=0.5$ can be directly obtained through the viral expansion of the local Hamiltonian if the polymer-solvent interaction is parameterized by the Flory $\chi$ parameter, the two methods we present in this paper, that is tracking the chain-length dependence of the melting of the globule and tracking the chain-length dependence of the solubility of polymer solution, are more general approaches in determining the $\Theta$ point. For example, the approaches can also be used for heteropolymers. Moreover, the consistency of the $\Theta$ point defined by the different criteria allows us to choose a convenient way to measure the $\Theta$ point in experiment. Our results suggest that the $\Theta$ point can be determined by measuring the slope of $\ln \phi_{L}$ versus $N^{2 / 3}$ for different temperatures, which is a finite-concentration instead of a single-chain measurement. 
1 Flory, P. J. Principles of Polymer Chemistry; Cornell University Press: Ithaca, NY, 1953.

2 des Cloizeaux, J.; Jannink, G. Polymers in Solution. Their modelling and Structure; Clarendon Press: Oxford, 1990.

3 de Gennes, P. G. Scaling Concept in Polymer Physics; Cornell University Press: Ithaca, NY, 1993.

4 Freed, K. F. Renormalization Group Theory of Macromolecules; Wiley: New York, 1987.

5 Yamakawa, H. Modern Theory of Polymer Solutions; Harper Row: New York, 1971.

6 Rubinstein, M.; Colby, R. H. Polymer Physics; Oxford University Press: Oxford, 2003.

7 Fujita, H. Polymer Solutions; Elsevier: New York,1990.

8 Takano, N.; Einaga, Y.; Fujita, H. Polym. J. 1985, 17, 1123-1130.

9 Dobashi, T.; Nakata, M.; Kaneko, M. J. Chem. Phys. 1980, 72, 6692-6697.

10 Madden, W. G.; Pesci, A. I.; Freed, K. F. Macromolecules 1990, 23, 1181-1191.

11 Yan, Q. Y.; Liu, H. L.; Hu, Y. Macromolecules 1996, 29, 4066-4071.

12 Panagiotopoulos, A. Z.; Wong, V.; Floriano, M. A. Macromolecules 1998, 31, 912-918.

13 Grossberg, A. Y.; Khokhlov, A. R. Statistical Physics of Macromolecules; AIP Press: New York 1994.

14 Wang, R.; Wang, Z. -G. Macromolecules 2012, 45, 62666217.

15 Nishio, I.; Sun, S. T.; Swislow, G.; Tanaka, T. Nature 1979, 281, 208-209.

16 Swislow, G.; Sun, S. T.; Nishio, I.; Tanaka, T. Phys. Rev. Lett. 1980, 44, 796-798.

17 Wu, C.; Wang, X. H. Phys. Rev. Lett. 1998, 80, 4092-4094.

18 Wang, X. H.; Chou, X. P.; Wu, C. Macromolecules 1998, 31, 2972-2976.

19 Dai. Z. J.; Wu, C. Macromolecules 2010, 43, 10064-10070.

20 Baysal, B. M.; Karasz, F. E. Macromol. Theory Simul. 2003, 12, 627-646.

${ }^{21} \mathrm{Hu}, \mathrm{W}$. J. Chem. Phys. 1998, 109, 3686-3690.

${ }^{22}$ Milchev, A.; Paul, W.; Binder, K. J. Chem. Phys. 1993, 99, 4786-4798.

23 Yang, D. L.; Wang, Q. ACS Macro Lett. 2013, 2, 952-954.

24 Williams, C.; Brochard, F.; Frich, C. H. Ann. Rev. Phys. Chem. 1981, 32, 433-451.

${ }^{25}$ Lifshitz, I. M.; Grossberg, A. Y.; Khokhlov, A. R. Reu. Mod. Phys. 1978, 50, 683-713.

${ }^{26}$ Khokhlov, A. R.; Khalatur, P. G. Phys. Rev. Lett. 1999, 82, 3456-3459.

27 Shakhnovich, E. Chem. Rev. 2006, 106, 1559-1588.

28 Gelbart, W. M.; Bruinsma, R. F.; Pincus, P. A.; Parsegian, V. A. Phys. Today 2000, 53, 38-44.

29 Suzuki, A.; Tanaka, T. Nature 1990, 346, 345-347.

30 Xiang, M. L. ; Jiang, M.; Zhang, Y. B.; Wu, C.; Feng, L. X. Macromolecules 1997, 30, 2313-2319.

31 de Gennes, P. G. J. Phys. (Paris) 1975, 36, 55.

32 Grassberger, P.; Hegger, R. J. Chem. Phys. 1995, 102,
6881-6899

33 Withers, I. M.; Dobrynin, A. V.; Berkowitz, M. L.; Rubinstein, M. J. Chem. Phys. 2003, 118, 4721-4732.

${ }^{34}$ Grosberg, A. Yu.; Kuznetsov, D. V. Macromolecules 1992, 25, 1970-1979.

35 Grosberg, A. Yu.; Kuznetsov, D. V. Macromolecules 1992, 25, 1980-1990.

36 Grosberg, A. Yu.; Kuznetsov, D. V. Macromolecules 1992 , 25, 1991-1995.

37 Grosberg, A. Yu.; Kuznetsov, D. V. Macromolecules 1992, 25, 1996-2003.

38 Ptitsyn, O. B.; Kron, A. K.; Eizner, Y. Y. J. Polym. Sci., Part C: Polym. Symp. 1968, 16, 3509.

39 Birshtein, T. M.; Pryamitsyn, V. A. Macromolecules 1991, 24, 1554

40 Bruns, W. Macromolecules 1984, 17, 2826-2830.

41 Sheng, Y. J.; Panagiotopoulos, A. Z. Kumar, S. K.; Szleifer, I. Macromolecules 1994, 27, 400-406.

42 Witelski, T. P.; Grosberg, A. Y.; Tanaka, T. J. Chem. Phys 1998, 108, 9144-9149.

43 Wang, J.; Guo, K.; An, L.; Müller, M.; Wang, Z.-G. Macromolecules 2010, 43, 2037-2041.

44 Wood, S. M.; Wang, Z.-G. J. Chem. Phys 2002, 117, 22892300.

45 Fredrickson, G. H. The Equilibrium Theory of Inhomogeneous Polymers; Oxford University Press: Oxford, 2006.

46 Fredrickson, G. H.; Ganesan, V.; Drolet, F. Macromolecules 2002, 35, 16-39.

47 Equation 3 is solved by using the implicit Crank-Nicolson method with 2000 grid points along the chain length and 2000 grid points along the spherical radius.

48 Wang, Z. -G. J. Chem. Phys 2002, 117, 481-500.

49 Qin, J.; Morse, D. C. Phys. Rev. Lett. 2012, 108, 238101.

50 We note that in our previous work ${ }^{14}$, the equation (Eq. 2 in that reference) incorrectly contains an extra $-N^{-1} \rho_{0}$ term. However, that equation was not used for any of the actual calculations, so the results and conclusions in Ref. ${ }^{14}$ are unaffected by this error.

51 Dobrynin, A. V.; Rubinstein, M. Macromolecules 1999, 32, 915-922.

52 This can be easily shown by assuming the density profile to be a flat region plus a decaying function of range $h_{T}$.

53 Zhou, Y. Q.; Hall, C. K.; Karplus, M. Phys. Rev. Lett. 1996, 7r, 2822-2825.

54 Szleifer, I.; Widom, B. J. Chem. Phys. 1989, 90, 75247534 .

${ }^{55}$ Weber, T. A.; Helfand, E. Macromolecules 1976, 9, 311316.

56 Strictly speaking, the critical point corresponds to $\partial \phi_{L} / \partial \chi \rightarrow \infty$. However, since our method is only valid in the swollen globule regime, we are unable to reach the critical point. Therefore, rather than a divergence, we obtain a discontinuity. 